\title{
Study of the Effective Pair Interatomic Interaction in a Co-Ni Melt in Terms of the Wills-Harrison Model
}

\author{
N. E. Dubinin \\ Ural Federal University, ul. Mira 19, Yekaterinburg, 620002 Russia \\ e-mail:ned67@mail.ru \\ Received November 8, 2012
}

\begin{abstract}
The dependence of the effective partial Wills-Harrison pair potentials in the equiatomic $\mathrm{Co}-\mathrm{Ni}$ melt near the melting temperature on the probability of the existence of both diagonal and nondiagonal (in magnetic quantum number) overlappings of $d$ electrons located at different atoms in the molten metal is analyzed.
\end{abstract}

DOI: $10.1134 / \mathrm{S} 0036029513020158$

\section{INTRODUCTION}

The model proposed by Wills and Harrison (WH) [1] to calculate the internal energy and the effective pair interatomic interaction in transition metals is widely used to study transition-metal melts [2-8].

In [9], we suggested a corrected WH model: the effective $d-d$ electron-electron interaction in a metal takes into account the contributions caused by the nondiagonality in a magnetic quantum number.

Since the degree of this nondiagonality is unknown, we introduced the probability $p$ of the existence of nondiagonal $d-d$ electron overlappings between two atoms in a metal provided they are equally probable between each other and with diagonal overlappings. Then, the WH model that takes into account only five equally probable diagonal $d-d$ overlappings is valid at $p=0$, and the fully corrected $\mathrm{WH}$ model with 25 equally probable (5 diagonal and 20 nondiagonal) overlappings holds true at $p=1$.

In this work, we apply this correction to calculate partial Wills-Harrison pair potentials for the equiatomic $\mathrm{Co}-\mathrm{Ni}$ melt and analyze how a change in $p$ influences their characteristics.

\section{THEORY}

The partial WH pair potential for a binary system $\varphi_{i j \mathrm{WH}}(r)$ is written as [7]

$$
\varphi_{i j \mathrm{WH}}(r)=\varphi_{s i j}(r)+\varphi_{d i j}(r),
$$

where $\varphi_{s i j}(r)$ and $\varphi_{d i j}(r)(i, j=1,2)$ are the contributions induced by the $s$ and $d$ electron states, respectively.
Contribution $\varphi_{s i j}(r)$ is calculated in terms of the second order of the perturbation theory in pseudopotential (hereafter, in atomic units (a.u.)),

$$
\varphi_{s i j}(r)=\frac{z_{s i} z_{s j}}{r}+\frac{1}{8 \pi^{2} \rho} \int_{0}^{\infty} F_{i j}(q) \frac{\sin (q r)}{q r} q^{2} \mathrm{~d} q,
$$

where $\rho$ is the mean atomic density, $z_{s i}$ is the effective $s$ electron valence of a pure metal of the $i$ th kind, and $F_{i j}(q)$ is the partial characteristic function

$$
F_{i j}(q)=-\frac{q^{2} \omega_{i}(q) \omega_{j}(q)}{\pi \rho\left[(\varepsilon(q)-1)^{-1}+1-f(q)\right]} .
$$

Here, $\varepsilon(q)$ is the Hartree dielectric function, $f(q)$ is the exchange-correlation function taken in the VashishtaSingwi approximation [10], and $\omega_{i}(q)$ is the form factor of the pseudopotential of an unscreened ion of the $i$ th kind in the binary system.

Following [6], we use the local Brettonet-Silbert (BS) model pseudopotential [11] generalized to binary systems in [12],

$$
\omega_{i \mathrm{BS}}(r)=\left\{\begin{array}{l}
\sum_{n=1}^{2} B_{n i} \exp \left(\frac{r}{n a_{i}}\right), \quad r \leq R_{\mathrm{C} i}, \\
-z_{s i} / r, \quad r \geq R_{\mathrm{C} i}
\end{array}\right.
$$

where $R_{C i}$ and $a_{i}$ are the BS model pseudopotential parameters and $B_{1 i}$ and $B_{2 i}$ are the coefficients found from the condition of continuity of $\omega_{i \mathrm{BS}}(r)$ and its first derivative at $r=R_{C i}$,

$$
\begin{aligned}
& B_{1 i}=\left(z_{s i} / R_{\mathrm{C} i}\right)\left(1-2 a_{i} / R_{\mathrm{C} i}\right) \exp \left(R_{\mathrm{C} i} / a_{i}\right), \\
& B_{2 i}=\left(2 z_{s i} / R_{\mathrm{C} i}\right)\left(a_{i} / R_{\mathrm{C} i}-1\right) \exp \left(0.5 R_{\mathrm{C} i} / a_{i}\right) .
\end{aligned}
$$


Input data used for the calculation

\begin{tabular}{c|c|c|c|c|c|c}
\hline Metal & $z_{s i}$ & $z_{d i}$ & $r_{d i}$ (a.u.) & $R_{C i}$ (a.u.) & $a_{i}$ (a.u.) & $\rho$ (a.u.) \\
\hline Co & 1.4 & 7.6 & 1.437 & 1.641 & 0.393 & 0.011648 \\
$\mathrm{Ni}$ & 1.4 & 8.6 & 1.342 & 1.030 & 0.207 & 0.011732 \\
\hline
\end{tabular}

Form factor $\omega_{i \mathrm{BS}}(q)$ is determined from the expression

$$
\begin{aligned}
\omega_{i \mathrm{BS}}(q)= & 4 \pi \rho a_{i}^{3}\left[\frac{B_{1 i} J_{1 i}(q)}{\left(1+a_{i}^{2} q^{2}\right)^{2}}+\frac{8 B_{2 i} J_{2 i}(q)}{\left(1+4 a_{i}^{2} q^{2}\right)^{2}}\right] \\
& -\left(4 \pi \rho z_{s i} / q^{2}\right) \cos \left(q R_{\mathrm{C} i}\right),
\end{aligned}
$$

where

$$
\begin{gathered}
J_{1 i}(q)=2-\exp \left(-\frac{R_{\mathrm{C} i}}{a_{i}}\right)\left\{\left[R_{\mathrm{C} i} \frac{1+a_{i}^{2} q^{2}}{a_{i}}+1-a_{i}^{2} q^{2}\right]\right. \\
\left.\times \frac{\sin \left(q R_{\mathrm{C} i}\right)}{a_{i} q}+\left[2+\frac{R_{\mathrm{C} i}\left(1+a_{i}^{2} q^{2}\right)}{a_{i}}\right] \cos \left(q R_{\mathrm{C} i}\right)\right\} ; \\
J_{2 i}(q)=2-\exp \left(-\frac{R_{\mathrm{C} i}}{2 a_{i}}\right)\left\{\left[R_{\mathrm{C} i} \frac{1+4 a_{i}^{2} q^{2}}{2 a_{i}}+1-4 a_{i}^{2} q^{2}\right]\right. \\
\left.\times \frac{\sin \left(q R_{\mathrm{C} i}\right)}{2 a_{i} q}+\left[2+\frac{R_{\mathrm{C} i}\left(1+4 a_{i}^{2} q^{2}\right)}{2 a_{i}}\right] \cos \left(q R_{\mathrm{C} i}\right)\right\} .
\end{gathered}
$$

Partial pair potential $\varphi_{d i j}(r)$ entering in the righthand side of Eq. (1) consists of the following two parts:

$$
\varphi_{d i j}(r)=\varphi_{b i j}(r)+\varphi_{c i j}(r),
$$

where $\varphi_{b i j}(r)$ is the contribution caused by the presence of a $d$ band with a finite width in the metal and $\varphi_{c i j}(r)$ is the contribution induced by the displacement of the center of the $d$ band due to the nonorthogonality of the $d$ states in the metal. We have

$$
\begin{gathered}
\varphi_{b i j}(r)=-\bar{z}_{d}\left(\frac{10-\bar{z}_{d}}{10}\right)\left(\frac{12}{v_{i j}}\right)^{1 / 2} \frac{\left(r_{d i} r_{d j}\right)^{3 / 2}}{r^{5}} K_{b}, \\
\varphi_{c i j}(r)=\bar{z}_{d} \frac{\left(r_{d i} r_{d j}\right)^{3}}{r^{8}} K_{c} .
\end{gathered}
$$

Here, $\bar{z}_{d}=c_{1} z_{d 1}+c_{2} z_{d 2}\left(z_{d i}\right.$ is the effective $d$ electron valence of the pure metal of the $i$ th kind, $c_{i}$ is the concentration of the component of the $i$ th kind in the binary system), $r_{d i}$ is the parameter having the physical meaning of the radius of the $d$ state of the free atom of the $i$ th kind, $v_{i j}$ is the partial coordination number, and
$K_{b}$ and $K_{c}$ are the coefficients written in the form proposed in [9]. They are

$$
\begin{gathered}
K_{b}=\left[\frac { 1 } { 5 } \left(\left(1-\frac{4 p}{5}\right) y_{0}^{2}+\left(2-\frac{6 p}{5}\right)\left(y_{2}^{2}+y_{1}^{2}\right)\right.\right. \\
\left.\left.+\frac{4 p}{5} y_{0}\left(y_{1}+y_{2}\right)+\frac{8 p}{5} y_{1} y_{2}\right)\right]^{\frac{1}{2}}, \\
K_{c}=-\frac{2}{5}\left[\left(1-\frac{4 p}{5}\right) y_{0} x_{0}+\left(2-\frac{6 p}{5}\right)\left(y_{1} x_{1}+y_{2} x_{2}\right)\right. \\
\left.+\frac{2 p}{5}\left(y_{0}\left(x_{1}+x_{2}\right)+x_{0}\left(y_{1}+y_{2}\right)\right)+\frac{4 p}{5}\left(y_{1} x_{2}+y_{2} x_{1}\right)\right],
\end{gathered}
$$

where $y_{m}=y_{|m|}$ and $x_{m}=x_{|m|}$ are the coefficients depending on quantum number $m$,

$$
\begin{aligned}
& y_{m}=-\frac{(-1)^{m} 180}{\pi(2+m) !(2-m) !}, \\
& x_{m}=-\frac{1}{8}\left(1+\frac{4 m^{2}-1}{9}\right) y_{m} .
\end{aligned}
$$

\section{RESULTS AND DISCUSSION}

The WH partial pair potentials in the equiatomic Co-Ni melt were calculated near the melting temperature at various values of $p$.

The table gives $r_{d i}$ and $z_{s i}$ taken from [1] and [13], respectively, and $z_{d i}=z_{i}-z_{s i}$ (where $z_{i}$ is the total valence of the pure metal of the $i$ th kind) and the BS model pseudopotential parameters borrowed from [13], where they were determined for pure liquid metals. The mean atomic density of a binary melt is taken to be additive with respect to the corresponding values of $\rho$ of the pure metals (which were experimentally obtained in [14] at $T=1823 \mathrm{~K}$ for Co and at $T=$ $1773 \mathrm{~K}$ for $\mathrm{Ni}$ ), which is allowable due to the closeness of these values (see table). The values of all three $v_{i j}$ are assumed to be 12 .

The obtained pair potentials are shown in the figure. As in the case of pure metals [9], an increase in $p$ for all potentials decreases the depth of the first minimum of the pair potential, weakly affects its coordinate when $p$ changes from 0 to 0.5 , and significantly shifts its position toward large $r$ at $p=1$ as compared to the case at $p=0.5$.

It was also found that the ordering potential $V=$ $\varphi_{12}(r)-\left(\varphi_{11}(r)+\varphi_{22}(r)\right) / 2$ at $p=0$ and $p=0.5$ is close to zero $(V \approx 0)$, which indicates almost ideal mixing of the components, whereas a tendency toward separation $(V>0)$ takes place at $p=1$.

The tendency toward separation in the liquid phase is unlikely in the $\mathrm{Co}-\mathrm{Ni}$ system, since it follows from the $\mathrm{Co}-\mathrm{Ni}$ phase diagram [15] that $\mathrm{Co}$ and $\mathrm{Ni}$ are infinitely dissolved in each other in the crystalline state and form a continuous series of solid solutions. Thus, the result obtained can indicate that the nondiagonal $d-d$ overlappings between different atoms do not gen- 

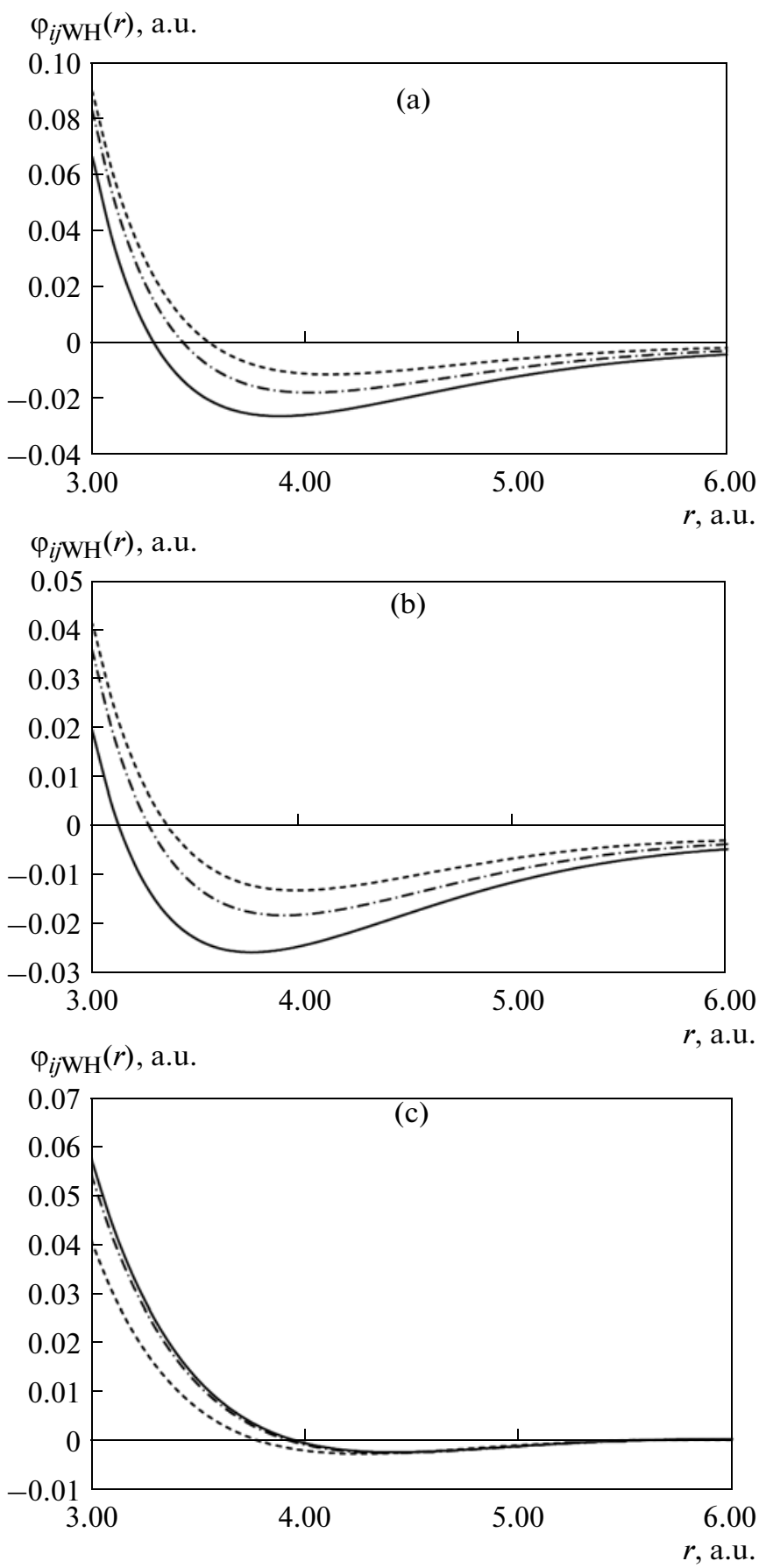

Partial WH pair potentials in the equiatomic Co-Ni melt near the melting temperature: (solid line) $\varphi_{\mathrm{Co}-\mathrm{Co}}(r)$, (dot-and-dash line) $\varphi_{\mathrm{Co}-\mathrm{Ni}}(r)$, and (dashed line) $\varphi_{\mathrm{Ni}-\mathrm{Ni}}(r)$ at $p=$ (a) 0 , (b) 0.5 , and (c) 1 .

erate a predominant contribution to the effective $d-d$ electron interaction in the liquid metal.

\section{ACKNOWLEDGMENTS}

This work was supported by the basic research program of the Ural Branch of the Russian Academy of Sciences, project no. 12-T-3-1022.

\section{REFERENCES}

1. J. M. Wills and W. A. Harrison, "Interionic interactions in transition metals," Phys. Rev. B 28, 4363-4373 (1983).

2. C. Hausleitner and J. Hafner, "Soft-sphere reference system in thermodynamic variational calculations. II: Liquid transition metals,” J. Phys. F: Metal Phys. 18, 1025-1035 (1988). 
3. C. Regnaut, "Analysis of the liquid structure of $3 d$ transition metals from the Wills-Harrison model," Z. Phys. B 76, 179-184 (1989).

4. J. L. Bretonnet and A. Derouiche, "Variational thermodynamic calculations for liquid transition metals," Phys. Rev. B 43, 8924-8929 (1991).

5. C. Hausleitner, G. Kahl, and J. Hafner, "Liquid structure of transition metals: investigations using molecular dynamics and perturbation- and integral-equation techniques," J. Phys. F: Condens. Matter 3, 15891602 (1991).

6. N. E. Dubinin, L. D. Son, and N. A. Vatolin, "The Wills-Harrison approach to the thermodynamics of binary liquid transition-metal alloys," J. Phys. F: Condens. Matter 20, 114111 (2008).

7. N. E. Dubinin, "Thermodynamics of liquid $\mathrm{Fe}-\mathrm{Ni}$ alloys: calculations at different temperatures," J. Phys.: Conf. Series, 144, 012115 (2009).

8. N. E. Dubinin, "Interatomic pair interaction in liquid transition metals," Acta Physica Polonica 115 (4), 783-785 (2009).

9. N. E. Dubinin, "Account of non-diagonal coupling between $d$ electrons at describing the transition-metal pair potentials," J. Phys.: Conf. Series 338, 012004 (2012).

10. P. Vashishta and K. Singwi, "Electron correlation at metallic densities," Phys. Rev. B 6, 875-887 (1972).

11. J. L. Bretonnet and M. Silbert, "Interionic interactions in transition metals. Application to vanadium," Phys. Chem. Liq. 24, 169-176 (1992).

12. N. E. Dubinin, L. D. Son, and N. A. Vatolin, "Thermodynamic properties of liquid binary transition-metal alloys in the Bretonnet-Silbert model," Defect and Diffusion Forum 263, 105-110 (2007).

13. N. Jakse and J. L. Bretonnet, "Structure and thermodynamics of liquid transition metals: integral-equation study of Fe, Co and Ni," J. Phys.: Condens. Matter 7, 3803-3815 (1995).

14. Y. Waseda, The Structure of non-Crystalline MaterialsLiquids and Amorphous Solids (McGraw-Hill, New York, 1981).

15. Phase Diagrams of Binary Metallic Systems: a Handbook, Ed. by N. P. Lyakishev (Mashinostroenie, Moscow, 1997), Vol. 2.

Translated by K. Shakhlevich 